\title{
EFFECT OF LOCAL TRANEXAMIC ACID ON THE QUALITY OF BONE HEALING IN A RAT SPINAL FUSION MODEL
}

Ertugrul Sahin ${ }^{1}$, Haluk Berk ${ }^{2}$, Sermin Ozkal $^{3}$, Pembe Keskinoglu ${ }^{4}$, Pinar Balci ${ }^{5}$, Ali Balci ${ }^{5}$

${ }^{1}$ Department of Orthopaedics and Traumatology, Kemalpasa State Hospital, Izmir, Turkey.

${ }^{2}$ Department of Orthopaedics and Traumatology, Dokuz Eylul University School of Medicine, Izmir, Turkey.

${ }^{3}$ Department of Pathology, Dokuz Eylul University School of Medicine, Izmir, Turkey.

${ }^{4}$ Department of Biostatistics and Informatics Dokuz Eylul University School of Medicine, Izmir, Turkey

${ }^{5}$ Department of Radiology, Dokuz Eylul University School of Medicine, Izmir, Turkey.

\section{Corresponding Author: Ertugrul Sahin, MD}

Department of Orthopaedics and Traumatology Kemalpasa State Hospital, Sekiz Eylul Mahallesi, Kırovası küme boulevard, 8/1 street, Post Zip Code: 35730, Kemalpaşa, Izmir, Turkey, Phone +90 50680822 69, Fax +90 232 4122639 , ertugrulsahinn@hotmail.com

\section{Reporting Checklist}

The authors have completed the ARRIVE reporting checklist.

\section{Funding}

This research received no specific grant from any funding agency in the public, commercial, or not-for-profit sectors.

\section{Conflicts of Interest}

All authors have completed the ICMJE uniform disclosure form. The authors have no conflicts of interest to declare. 


\section{Ethics approval}

Dokuz Eylül University Multidisciplinary Laboratory Animal Experiments Local Ethics Committee approved this research with protocol number 19/2019 on May 8, 2019.

\section{Informed Consent}

Consent was not required because this study involved no human subject.

\section{Acknowledgments}

Thanks to my wife, Gonca SAHIN MD, for her support and time for this study. Also, thanks to Toklong Filam Moses MD, Orkun Halac MD, and Berkay Yanik MD who evaluated the motion of fusion level and scores.

\section{Author's Contribution}

(I) Conception and design: E. Sahin, H. Berk

(II) Administrative support: None

(III) Provision of study materials or patients: All authors

(IV) Collection and assembly of data: All authors

(V) Data analysis and interpretation: E. Sahin, H. Berk, and P. Keskinoglu

(VI) Manuscript writing: All authors

(VII) Final approval of manuscript: All authors 


\section{RAT SPINAL FUSION MODEL}

\section{ABSTRACT}

Introduction: The use of the antifibrinolytic agent tranexamic acid has positive effects on bleeding control, but our knowledge is still limited regarding how fibrinolysis suppression changes the process of bone formation and the quality of bone. Because of the several side effects of systemic tranexamic acid, topical usage has been established in several procedures. This study aimed to investigate the effect of local tranexamic acid on vertebral fusion by using macroscopic, radiologic, and microscopic techniques. We also attempted to determine the safe dose range in case some doses had negative effects on fusion.

11 Methods: Twenty-eight Wistar albino rats underwent intertransverse fusion. All rats were randomized into four groups: groups treated with local tranexamic acid doses of $1 \mathrm{mg} / \mathrm{kg}$ (D1), $10 \mathrm{mg} / \mathrm{kg}$ (D10), and $100 \mathrm{mg} / \mathrm{kg}$ (D100) and the control group with no drug (D0). At the end of the eighth week, all rats were sacrificed for

14 evaluation in terms of palpation, mammography, and histopathologic analysis.

15 Results: The manual palpation results presented with lower fusion rates in D10 and D100 groups than in the

16 control group. Radiological examination results were significantly higher in the control group. The

17 histopathologic examination revealed no significant differences between groups in the percent of new bone

18 formation.

19 Conclusion: Our results showed that local administration of tranexamic acid reduced the quality and stability of

20 fusion without a delay in bone formation. However, doses of $1 \mathrm{mg} / \mathrm{kg}$ did not reduce the stability in the palpation

21 test. Our findings suggest that $1 \mathrm{mg} / \mathrm{kg}$ dose is a critical threshold above which tranexamic acid reduced the bone healing process of fusion and that surgeons should consider the doses of local tranexamic acid during surgery.

23 Keywords Rat; Bone healing; Spinal fusion; Tranexamic acid; Antifibrinolytic; 
RAT SPINAL FUSION MODEL

\section{INTRODUCTION}

The use of an antifibrinolytic agent, tranexamic acid, has been shown to considerably reduce perioperative bleeding in several contexts, including cardiothoracic, orthopedic, and spinal surgeries ${ }^{1-3)}$.

Tranexamic acid inhibits the interaction of plasminogen and the heavy chain of plasmin with lysine residues on the surface of fibrin because of its high affinity for the lysine-binding site of plasminogen, thereby resulting in the inhibition of the formation of fibrin degradation products ${ }^{4}$. There are two ways to use tranexamic acid: systemically and locally. The systemic administration of tranexamic acid has some potential adverse effects in patients with deep vein thrombosis, pulmonary embolism, myocardial infarction, and seizures ${ }^{5-7)}$. These potential side effects have encouraged researchers to use local tranexamic acid for perioperative bleeding control.

Despite the positive effects of this drug on bleeding control, our knowledge is still limited regarding how fibrinolysis suppression changes the process of bone formation and the quality of bone. Fibrin matrices that form after fracture have essential factors for repair, and based on this information, most studies have reported the importance of fracture hematoma preservation in healing the bone ${ }^{8)}$. Additionally, a few studies have shown that a decrease in fibrinolysis promotes healing $\left.{ }^{9}, 10\right)$. Conversely, the accumulation of fibrin has negative effects on tissue repair and results in increased local inflammation, which could cause osteoporosis ${ }^{11)}$.

Pseudoarthrosis remains one of the most common reasons for revision surgery following spine fusion operations ${ }^{12}$. The most common factors associated with impaired fusion are smoking, infection, diabetes, vitamin D deficiency, impairment of bone formation, and advanced age ${ }^{13-16)}$. Moreover, all these factors are related to impaired fibrinolysis activity ${ }^{17,18)}$.

The process of bone fusion is a kind of wound healing and is expected to be affected by fibrinolytic coagulation proteases and their inhibitors ${ }^{19,20)}$. Our investigations have directed us to determine the effect of local tranexamic acid on spine fusion. We also aimed to determine the safe dose range in case some doses have negative effects on fusion.

\section{MATERIALS AND METHODS}

\section{Ethical Approval}


57 protocol was reviewed and approved by the multidisciplinary laboratory animal experiment local ethics

58 committee.

59

60

\section{Animals}

Healthy adult male Wistar albino rats weighing approximately $400 \mathrm{~g}$ and with normal motor activity were selected. All experiments were conducted at a temperature of approximately $20^{\circ} \mathrm{C}$ under a $12 \mathrm{~h}$ dark/light cycle. No feeding restrictions or changes were implemented.

\section{Experimental Design}

An ARRIVE checklist was used to ensure that the correct protocol had been applied. All rats were randomized into four groups by using sealed envelopes. Local tranexamic acid (Transamin $100 \mathrm{mg} / \mathrm{ml}$, Teva İlaç, İstanbul) were determined on the basis of previously conducted studies: $1 \mathrm{mg} / \mathrm{kg}$ (D1 group), $10 \mathrm{mg} / \mathrm{kg}$ (D10 group), $100 \mathrm{mg} / \mathrm{kg}$ (D100 group), and no tranexamic acid (D0 group) ${ }^{21)}$. Two rats in the D0 group died after the radiographic examination.

At 8 weeks postoperatively, plain radiographs were obtained. Then, specimens were prepared for manual palpation and histopathologic analysis. Palpation tests were performed immediately after sacrifice to reduce the effect of rigor mortis.

\section{Surgical Procedure}

After the rats were weighed, ketamine (Ketalar, Pfizer) $50 \mathrm{mg} / \mathrm{kg}$ and xylazine (Alfazyne, Egevet, İstanbul) $5 \mathrm{mg} / \mathrm{kg}$ were administered intraperitoneally for anesthesia. Preoperative ceftriaxone $50 \mathrm{mg} / \mathrm{kg}$ (Rocephin, Roche, İstanbul) was administered as prophylaxis $30 \mathrm{~min}$ before the incision was made ${ }^{22}$. The rats were positioned prone, and a dorsal midline skin incision between iliac crests was made. The transverse processes of the L4-L5 vertebrae were decorticated. Approximately $250 \mathrm{mg}$ of morselized autogenous bone grafts were harvested from the posterior parts of the iliac wings and placed into the fusion area (Figure 1). Tranexamic acid solution ( $1 \mathrm{~g}$ in $10 \mathrm{ml}$ of normal saline, prepared before the surgical procedure) was poured directly via injector to the fusion site according to dose ranges, given above. 


\section{Evaluation of Fusion}

At the end of the eighth week, sedated rats were positioned supine under mammography (Selenia ${ }^{\circledR}$ Dimensions ${ }^{\circledR}$ Mammography System, Hologic, Massachusetts, USA) (Figure 2). A previously described radiological scoring system was used to assess the radiographs (Table 1) ${ }^{23}$. All radiographs were evaluated blindly and scored by four researchers (two radiologists and two spine surgeons) twice 1 month apart on the Sectra Uniview digital imaging platform (version 21.2.11.6289, Linköping, Sweden). The first round scores were not planned to investigate for evaluation because we thought that the quality of images from mammography was not as high as micro-CT and there would be some difficulties to interpret them better. The second-round scores of each researcher were categorized. Radiographs were evaluated on the basis of the frequency of categorized scores.

All spines underwent palpation tests by three orthopedic surgeons immediately after sacrifice. Blinded evaluation of motion at the fusion level was performed utilizing a previously described fusion mass scoring system: "0" points, indicating the same range of motion as a nonfused level one below or one above the fusion; "1" point, indicating a decrease in the range of motion; and "2" points, indicating no motion ${ }^{24)}$. All values were categorized for each group and evaluated as the frequency of scores.

Each specimen underwent en bloc resection after manual palpation examination, and the specimens were then fixed in $10 \%$ formalin solution for $24 \mathrm{~h}$ and decalcified for $48-72 \mathrm{~h}$ in $20 \%$ formic acid. Four to eight consecutive sections with a thickness of 5-6 microns were generated by microtome and stained with hematoxylin and eosin. Heiple's modified Lane and Sandhu scoring ${ }^{25)}$ was used to determine histopathological changes in the cortical and trabecular bones and marrow areas (Table 2).

The distributions of the data were checked with the Kolmogorov-Smirnov normality test. Continuous variables are presented as the mean \pm standard deviation (SD). The differences were compared using one-way analysis of variance with Tukey's post hoc analysis for normally distributed data and the Kruskal-Wallis test for nonnormally distributed data. The Mann-Whitney U test was used for post hoc analysis with pairwise comparisons. Categorical data are presented as numbers and percentages (\%). Pearson's chi-square test was used to compare the fusion rates of each group. Spearman correlation coefficient analysis was used for the assessment 
of the intraobserver and interobserver correlation of the observer in radiological and palpation examinations. All analyses were performed using IBM SPSS Statistics 22 software. A p- value below 0.05 was accepted as statistically significant.

\section{RESULTS}

Twenty-eight rats were used in this study. The average weight of bone grafts for all groups was 249.08 $\pm 11.9 \mathrm{mg}$ with no statistical difference among all groups $(\mathrm{p}=0.22)$. In the control group, two rats died after

113 radiographic examination, likely because of anesthesia. The remaining 25 rats underwent all tests with no 114 complications.

Spearman's rho correlation coefficient was high in the palpation scoring $(\mathrm{r}=0.83)$. On the palpation test, the total scores of all observers were used to categorize spinal fusion as complete, partial, or absent and the distribution of fusion rates was shown in Figure 3. The rate of partially fused spines in the D0 group was 3 $(20 \%)$, that in the D1 group was $8(38.1 \%)$, that in the D10 group was $3(14.3 \%)$, and that in the D100 group was $3(14.3 \%)$. The rate of completely fused spines in the D0 group was $9(60 \%)$, that in the D1 group was 13

120 (61.9\%), that in the D10 group was 11 (52.4\%), and that in the D100 group was 7 (33.3\%). Statistically

121 significant differences in fusion rates were detected in all groups $(p=0.002)$. The rates of completely and partially fused spines were similar between the control and D1 groups $(p=0.32)$. Evaluation of fusion rates in

123 tranexamic-treated groups showed a decreasing trend with increasing dosage, and these differences were 124 significant $(\mathrm{p}=0.009)$.

Based on the radiological examination, Spearman's rho correlation coefficient was moderate in the first evaluation $(\mathrm{r}=0.46)$ and high in the second evaluation $(\mathrm{r}=0.61)$. All results of the second rounds were

127 categorized as complete, partial, or absent spinal fusion distribution of fusion rates, which are shown in Figure 4.

128 The rate of partially and completely fused spines in the D0 group was $96.4 \%$, that in the D1 group was $67.9 \%$, 129 that in the D10 group was $85.7 \%$, and that in the D100 group was $82.1 \%$. There was a significant difference 130 between groups $(\mathrm{p}=0.007)$. However, the evaluation of fusion rates in tranexamic-treated groups showed no 131 difference with changing dosages $(\mathrm{p}=0.27)$. 
133 Heiple's scoring system was applied, and all results are shown in Table 3. The total score was the highest in the

134 D1 group, followed by the D100, D0, and D10 groups. However, there was no statistically significant difference

135 in the mean values of the union, spongious, compact, or bone marrow subgroups or in the total scores $(\mathrm{p}=0.13)$.

136 The mean values of all subgroups in the tranexamic acid-treated groups have been evaluated; only the union

137 subgroups showed a difference $(p=0.04)$. The rate of union in the D1 group was higher than that in the D10

138 group (Dunn's multiple comparison test, $\mathrm{p}=0.03$ ).

\section{DISCUSSION}

In this study, our results indicated that the addition of tranexamic acid adversely affected the quality of fusion. The palpation test shows that this adverse effect was dependent on dosage but independent of radiographic examination. Histopathologic changes were similar in all groups.

To our knowledge, almost all studies on this topic have been about the effectiveness of tranexamic acid for bleeding control in spinal surgeries ${ }^{5}$. However, the effect of local tranexamic acid on fusion quality has not yet been investigated. In the literature, there were a few studies that investigated the effects of the fibrinolysis mechanism on bone union. Heather et al. ${ }^{11)}$ found that fibrinogen was the exogenous activator in bone remodeling and caused bone resorption leading to osteoporosis. Schoenecker et al. ${ }^{26)}$ found that although aminocaproic acid and tranexamic acid did not show any impact on mineralization with the treatment dosage, they decreased osteoblastic proliferation depending on the dosage. Yuasa et al ${ }^{8)}$ found that in cases where fibrinolysis deteriorated, the accumulation of fibrin deposits prevented the soft callus from transforming into a vascularized rigid callus. On the basis of these studies, we hypothesized that tranexamic acid would have a negative effect on vertebral fusion.

The evaluation time of fusion with manual palpation varies between 2 and 12 weeks, with an average of 1546 weeks. Fusion ranges were increased significantly in the eighth week ${ }^{27}$. Hence, we evaluated each specimen 8 weeks after the surgical procedure.

To avoid bias, we did not allocate rats into groups before the surgical procedure. Grouping of rats was

157 performed only after the bone graft was taken from the iliac wing, followed by the decortication of transverse 158 processes and placement of grafts into the fusion area. Afterward, the group of animals that underwent the fusion operation was decided according to a prerandomized sealed envelope. 

standard for the determination of fusion ${ }^{27,28)}$. Intervertebral movement of the spine with palpation is analogous to manual testing in humans during surgical exploration ${ }^{29}$. In our study, manual palpation was performed by three independent orthopedic surgeons who were blinded. We showed that the use of local $1 \mathrm{mg} / \mathrm{kg}$ tranexamic acid had no effect on fusion; however, increasing doses of tranexamic acid had a negative effect on fusion. examination for the evaluation of fusion has been used for a long time. In our study, radiological examination was performed only by direct graphics. Scoring was performed by two radiologists and two spinal surgeons. In our radiological imaging protocol, only the AP view was used because, in the lateral view, the vertebral body was projected on the intertransverse area and did not allow the assessment. In the first round, the interrater reliability was found to be moderate. In the second round of radiographic scoring, the control group had the best

172 outcomes, and local tranexamic acid adversely affected fusion regardless of the dose. Furthermore, interrater

173 correlation was found to be high. We attributed the difference between both scores to the image quality.

174 Evaluating radiographies twice provided the assessment of the intraobserver and interobserver differences.

175 Additionally, the increased correlation between researchers in the second round of evaluation led us to think that 176 the researchers adapted to the criteria of the scoring system and learned to interpret them better, although images 177 were presented in shuffled order with no definitive signs on them. Thus, we did not include the data of the first round examination. There is always a possibility of inconsistency between manual palpation and other

179 radiographic evaluations, and it will be necessary to define the fusion criteria to overcome this problem ${ }^{28,30)}$.

180 Consequently, radiological examination revealed that the control group had the best in fusion, and tranexamic acid adversely affected fusion, independent of dosage. Changing the dose did not show any significant effect.

183 decision is made by evaluating bone bridge formation. However, there is always a possibility of missing this

184 bone bridge in the slice taken. Bone healing and bone fusion are also dynamic and continuous processes, so the 185 histopathologic results are strongly dependent on the timing of sampling. Histopathologic scoring can only be 186 performed according to the stage of sampling time. Among the groups, no significant difference was found $187(\mathrm{p}=0.13)$. The results showed that tranexamic acid did not delay or accelerate fusion regardless of dose. 
The limitations of this study include its nature as an experimental study in a rat model. There are anatomical differences between humans and rats, and the speed of bone healing is faster in rats, which leads to less susceptibility to environmental factors. Because of these differences, tranexamic acid may affect the quality of vertebral fusion differently in rats and humans. Another limitation of our study was the use of mammography instead of micro-CT, considering its advantages. Radiographs were evaluated twice in 1 month by four researchers to strengthen the results and increase confidence. It should be kept in mind that this error may occur in all fusion studies that utilize manual examination. Future studies are needed to determine the impact of tranexamic acid on the quality of spine fusion by researching the molecular changes of bone graft substitutes through the fusion process. Additionally, clinical and radiographic follow-ups may be performed to investigate the effect of local tranexamic acid with different doses on vertebra fusion in humans. bone formation. There was no statistically significant difference in histopathological results between the groups. However, $1 \mathrm{mg} / \mathrm{kg}$ dose did not reduce the stability in the palpation examination. Our findings suggest that 1 $\mathrm{mg} / \mathrm{kg}$ dose might be a critical threshold above which tranexamic acid reduced the bone healing process of vertebra fusion and surgeons may consider the administration doses of local tranexamic acid during fusion surgery. Additionally, this study will be the reference for further studies on how tranexamic acid affects bleeding control at a dosage that does not affect fusion in the future.

\section{REFERENCES}

206 1. Myles PS, Smith JA, Forbes A, et al. Tranexamic acid in patients undergoing coronary-artery surgery. N 207 Engl J Med. 2017;376(2): 136-48.

208 2. Danninger T, Memtsoudis SG. Tranexamic acid and orthopedic surgery-the search for the Holy Grail of 209 blood conservation. Ann Transl Med. 2015;3(6): 77.

2103 3adeaux J, Hawley D. A systematic review of the effectiveness of intravenous tranexamic acid

211 administration in managing perioperative blood loss in patients undergoing spine surgery. J Perianesth Nurs.

$212 \quad 2014 ; 29(6): 459-65$.

2134 4unt BJ. The current place of tranexamic acid in the management of bleeding. Anaesthesia.

214 2015;70(suppl 1): 50-3, e18. 
215 5. Winter SF, Santaguida C, Wong J, et al. Systemic and topical use of tranexamic acid in spinal surgery:

216 A systematic review. Glob Spine J. 2016;6(3): 284-95.

217 6. Lecker I, Wang DS, Romaschin AD, et al. Tranexamic acid concentrations associated with human

218 seizures inhibit glycine receptors. J Clin Invest. 2012;122(12): 4654-66.

219 7. Ma TKW, Chow KM, Kwan BCH, et al. Manifestation of tranexamic acid toxicity in chronic kidney

220 disease and kidney transplant patients: a report of four cases and review of literature. Nephrology. 2017;22(4):

$221 \quad 316-321$.

222 8. Yuasa M, Mignemi NA, Nyman JS, et al. Fibrinolysis is essential for fracture repair and prevention of 223 heterotopic ossification. J Clin Invest. 2015;125(8): 3117-31.

224 9. Çevik HB, Eceviz E, Çilingir Kaya ÖT, et al. The effect of topical and systemic tranexamic acid on 225 fracture healing in rats. Acta Orthop Traumatol Turc. 2020;54(2): 207-12.

226 110. Karaduman ZO, Arıcan M. Turhan Y, et al. Systemic tranexamic acid promotes bone healing in a rat 227 model of femur fracture. Jt Dis Relat Surg. 2020;31(3): i-viii.

228 11. Cole HA, Ohba T, Nyman JS, et al. Fibrin accumulation secondary to loss of plasmin-mediated

229 fibrinolysis drives inflammatory osteoporosis in mice. Arthritis Rheumatol. 2014;66(8): 2222-33.

230 12. Martin BI, Mirza SK, Comstock BA, et al. Reoperation rates following lumbar spine surgery and the 231 influence of spinal fusion procedures. Spine. 2007;32(3): 382-7.

232 13. Brown CW, Orme TJ, Richardson HD. The rate of pseudarthrosis (surgical nonunion) in patients who 233 are smokers and patients who are nonsmokers: a comparison study. Spine. 1986;11(9): 942-3.

234 14. Ravindra VM, Godzik J, Dailey AT, et al. Vitamin D levels and 1-year fusion outcomes in elective 235 spine surgery: A prospective observational study. Spine. 2015;40(19): 1536-41.

236 15. Gruber R, Koch H, Doll BA, et al. Fracture healing in the elderly patient. Exp Gerontol. 2006;41(11): $237 \quad 1080-93$

238 16. Kline AJ, Gruen GS, Pape HC, et al. Early complications following the operative treatment of pilon 239 fractures with and without diabetes. Foot Ankle Int. 2009;30(11): 1042-47.

240 17. Meade TW, Chakrabarti R, Haines AP, et al. Characteristics affecting fibrinolytic activity and plasma 241 fibrinogen concentrations. Br Med J. 1979;1(6157): 153-6. 
242 18. Stępień E, Miszalski-Jamka T, Kapusta P, et al. Beneficial effect of cigarette smoking cessation on 243 fibrin clot properties. J Thromb Thrombolysis. 2011;32(2): 177-82.

244 19. Tumber A, Papaioannou S, Breckon J, et al. The effects of serine proteinase inhibitors on bone 245 resorption in vitro. J Endocrinol. 2003;178(3): 437-47.

24620.2 Daci E, Udagawa N, Martin TJ, et al. The role of the plasminogen system in bone resorption in vitro. J

247 Bone Miner Res. 1999;14(6): 946-52.

248 21. Schwarzkopf R, Dang P, Luu M, et al. Topical tranexamic acid does not affect electrophysiologic or 249 neurovascular sciatic nerve markers in an animal model. Clin Orthop Relat Res. 2015;473(3): 1074-82.

250 22. Yilmaz A, Karatay M, Yildirim T, et al. Prevention of epidural fibrosis using ranibizumab in a 251 postlaminectomy rat model. Turk Neurosurg. 2017;27(1): 119-123.

252 23. Curylo LJ, Johnstone B, Petersilge CA, et al. Augmentation of spinal arthrodesis with autologous bone 253 marrow in a rabbit posterolateral spine fusion model. Spine (Phila Pa 1976). 1999;24(5): 434-8.

254 24. Lane JM, Sandhu HS. Current approaches to experimental bone grafting. Orthop Clin North Am. $255 \quad 1987 ; 18(2): 213-25$.

256 25. Heiple KG, Goldberg VM, Powell AE, et al. Biology of cancellous bone grafts. Orthop Clin North Am. $257 \quad 1987 ; 18(2): 179-85$

258 26. Schoenecker J, Mignemi N, Stutz C, et al. 2010 young investigator award winner: therapeutic aprotinin 259 stimulates osteoblast proliferation but inhibits differentiation and bone matrix mineralization. Spine (Phila Pa 260 1976). 2010;35(9): 1008-16.

261 27. Ishida W, Elder BD, Holmes C, et al. Variables Affecting Fusion Rates in the Rat Posterolateral Spinal 262 Fusion Model with Autogenic/Allogenic Bone Grafts: A Meta-analysis. Ann Biomed Eng. 2016;44(11):3186263201

265 28. Grauer JN, Patel TC, Erulkar JS, et al. Evaluation of OP-1 as a graft substitute for intertransverse 266 process lumbar fusion. Spine (Phila Pa 1976). 2001;26(2): 127-33.

267 29. Yee AJM, Bae HW, Friess D, et al. Augmentation of rabbit posterolateral spondylodesis using a novel 268 demineralized bone matrix-hyaluronan putty. Spine (Phila Pa 1976). 2003;28(21): 2435-40. 

model. Radiographic, histologic, and biomechanical healing characteristics. Spine. 1995;20(4): 412-20.

\section{FIGURE CAPTIONS}

275 Figure 1. Placement of the bone grafts harvested from the posterior iliac wing to the intertransverse interval (the 276 grafts are shown by arrows).

277 Figure 2. Radiological examination of the rats: (A) control group, (B) D1 group, (C) D10 group, and (D) D100 group. Arrows indicate a fusion mass in the intertransverse field.

Figure 3. Fusion rates in manual palpation examination for all groups.

Figure 4. Fusion rates in the radiographic examination for all groups.

281 Figure 5. Specimens showing the formation of bone, cartilage, and marrow tissue in the intertrabecular fields

282 (H\&E, 40X). (A) Specimen with 4 points for union. The blue arrow demonstrates the field of chondrogenic

283 healing with the prominence of chondrocyte cells. Green arrows demonstrate trabecular bones, and hematopoietic cells become reorganized by gathering together. (B) Specimen with 3 points for union. The blue arrow demonstrates the field of chondrogenic healing with the prominence of chondrocyte cells. Black arrows indicate the new woven bone fields. The green arrow displays the fibrosis generated by the loose connective

287 tissues in the irregular trabecular bone fields that have gathered together. (C) Specimen with 1 point for union. Irregular bone formation (black arrows) and connective tissue containing dense fibrosis in intertrabecular fields (this area is shown by the blue arrow) (H\&E, 20X). Normal bone marrow containing hematopoietic cells (green arrow). (D) Specimen with 2 points for union. Blue arrows show dense cartilage tissue with chondrocytes. The area shown by the black arrow shows endochondral ossification, which is the beginning of bone formation. 


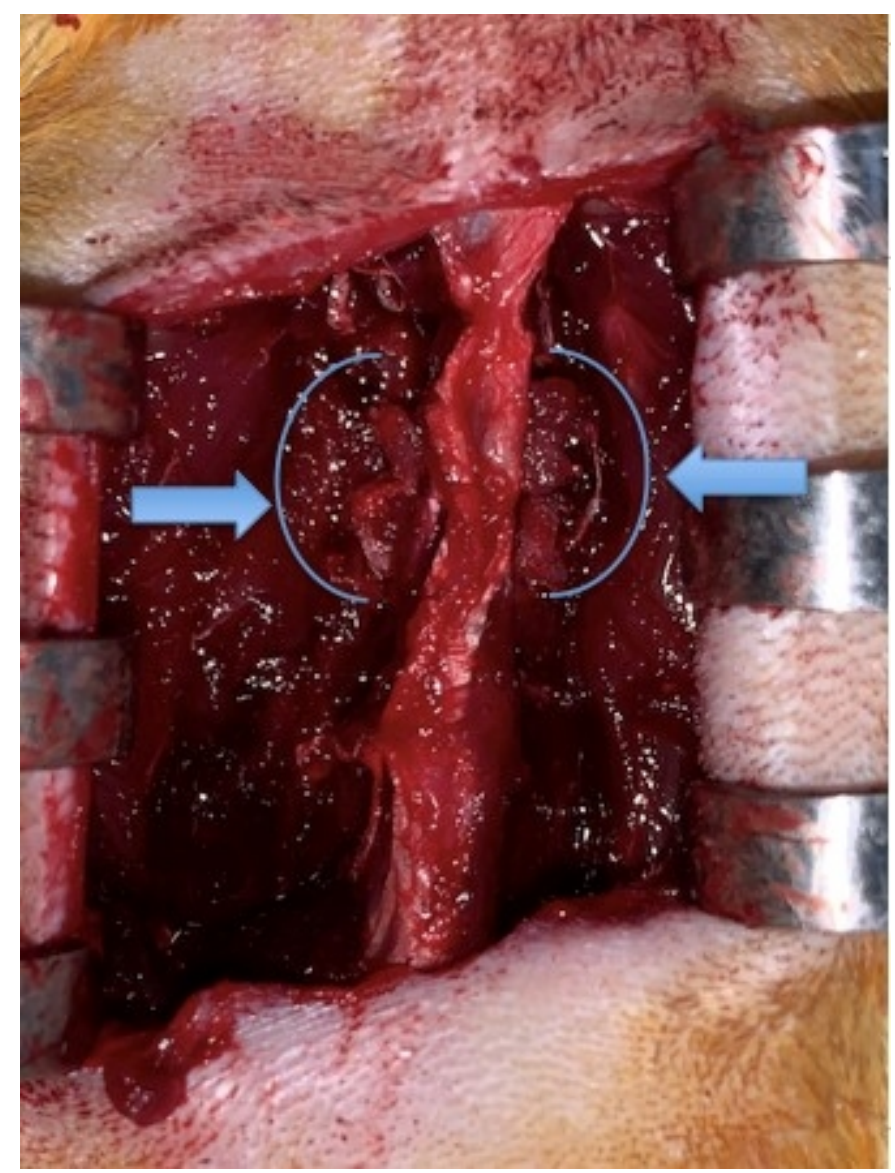

$88 \times 117 \mathrm{~mm}(96 \times 96 \mathrm{DPI})$ 


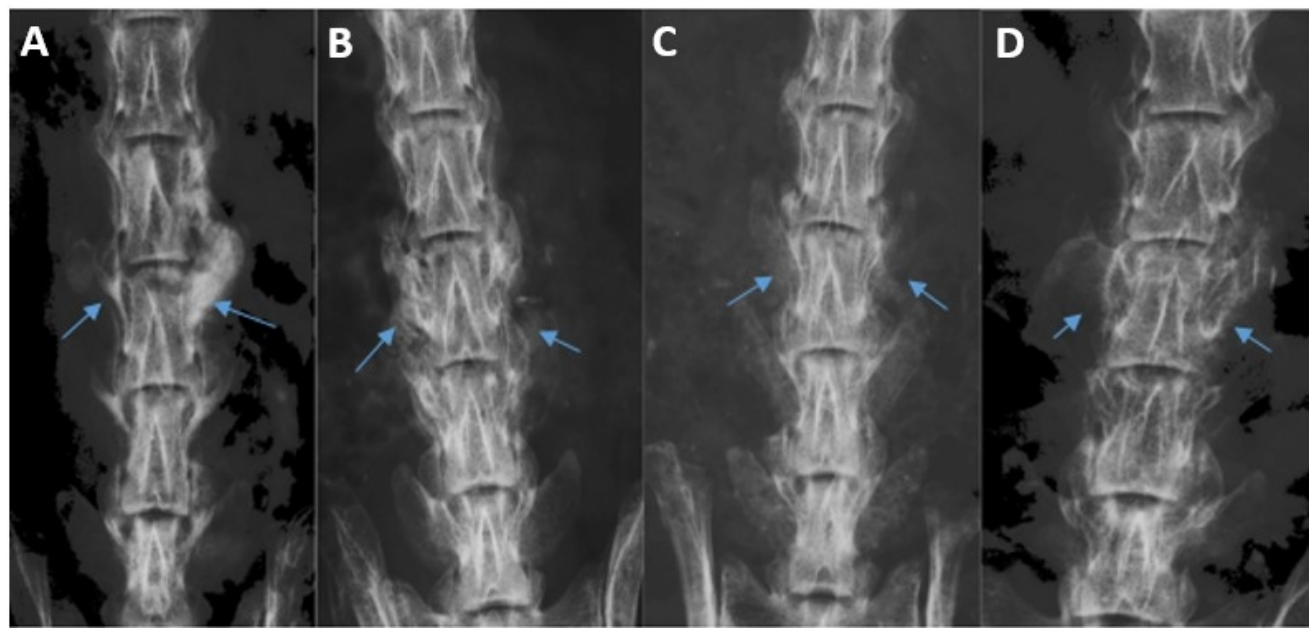

$209 \times 100 \mathrm{~mm}(96 \times 96$ DPI) 


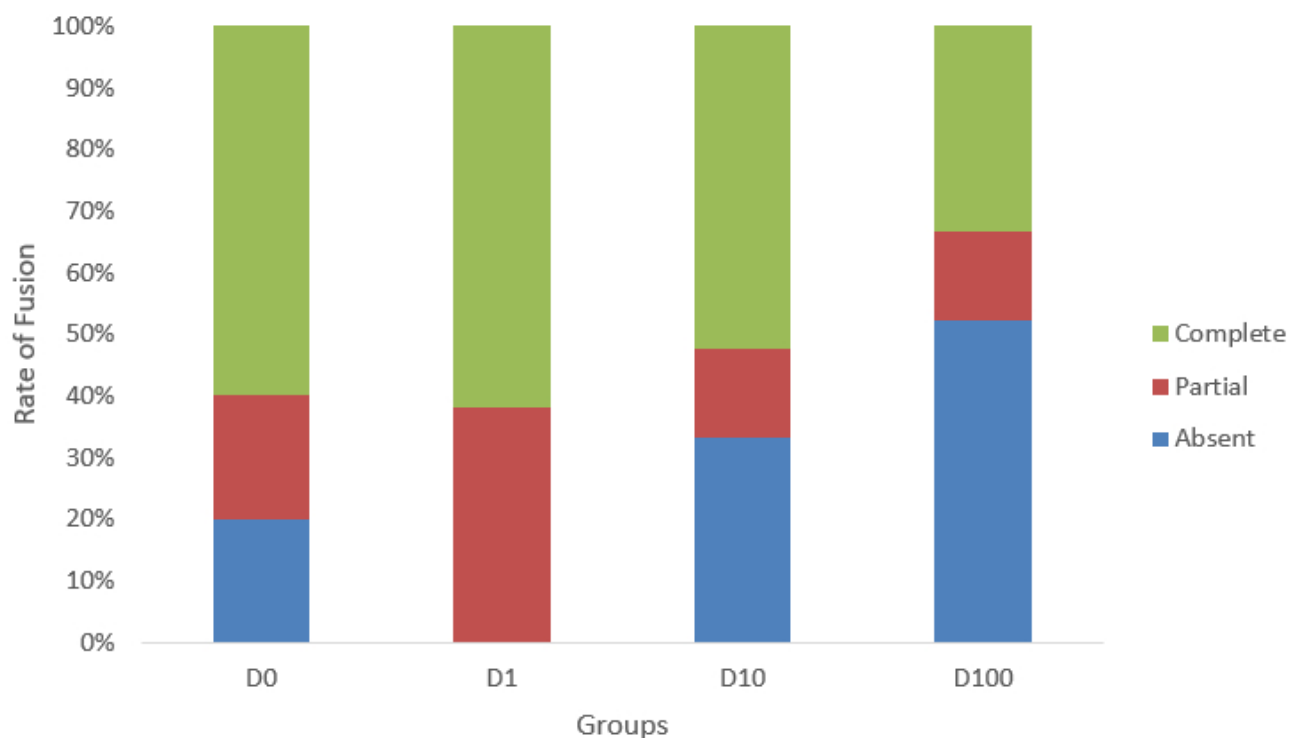

$396 \times 225 \mathrm{~mm}(47 \times 47 \mathrm{DPI})$ 


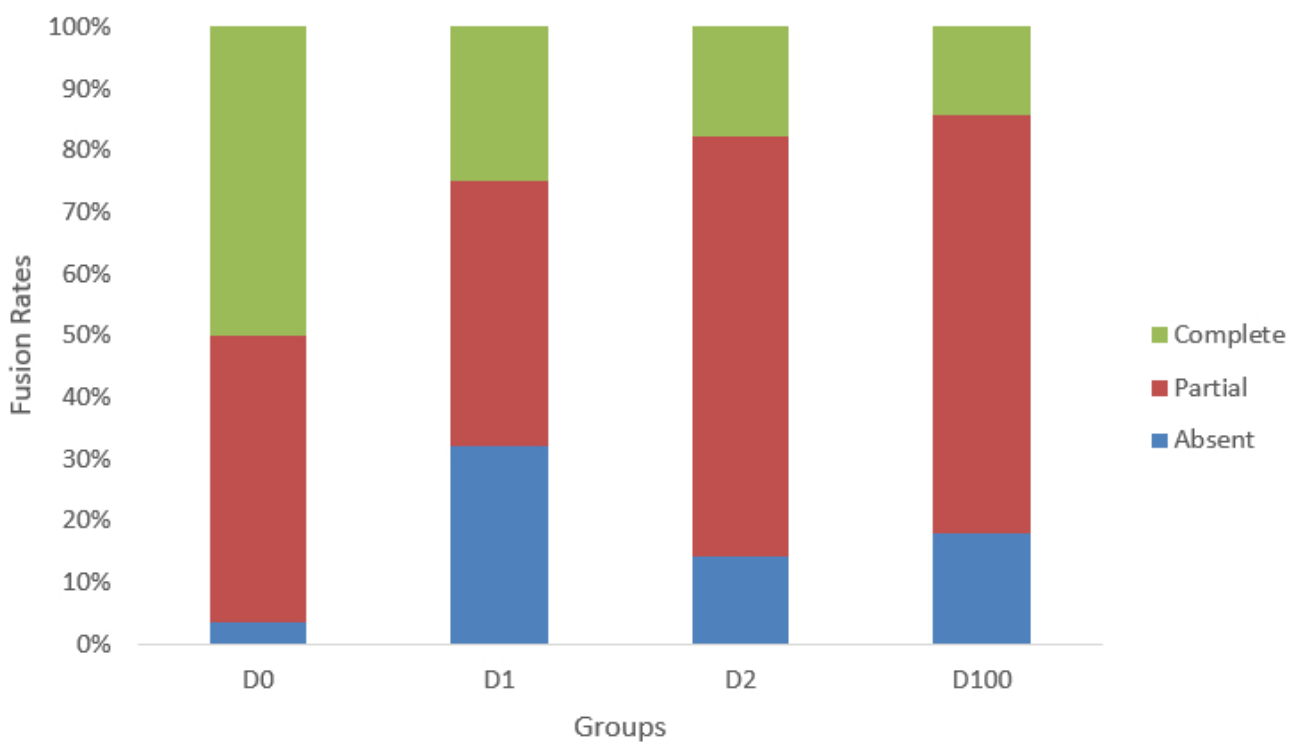

$395 \times 228 \mathrm{~mm}(47 \times 47$ DPI $)$ 
Spine Surgery and Related Research J-STAGE Advance Publication (October 11, 2021)

(C) The J apanese Society for Spine Surgery and Related Research. CC BY- NC- ND 4.0 (https://creativecommons.org/licenses/by- nc- nd/4.0/).
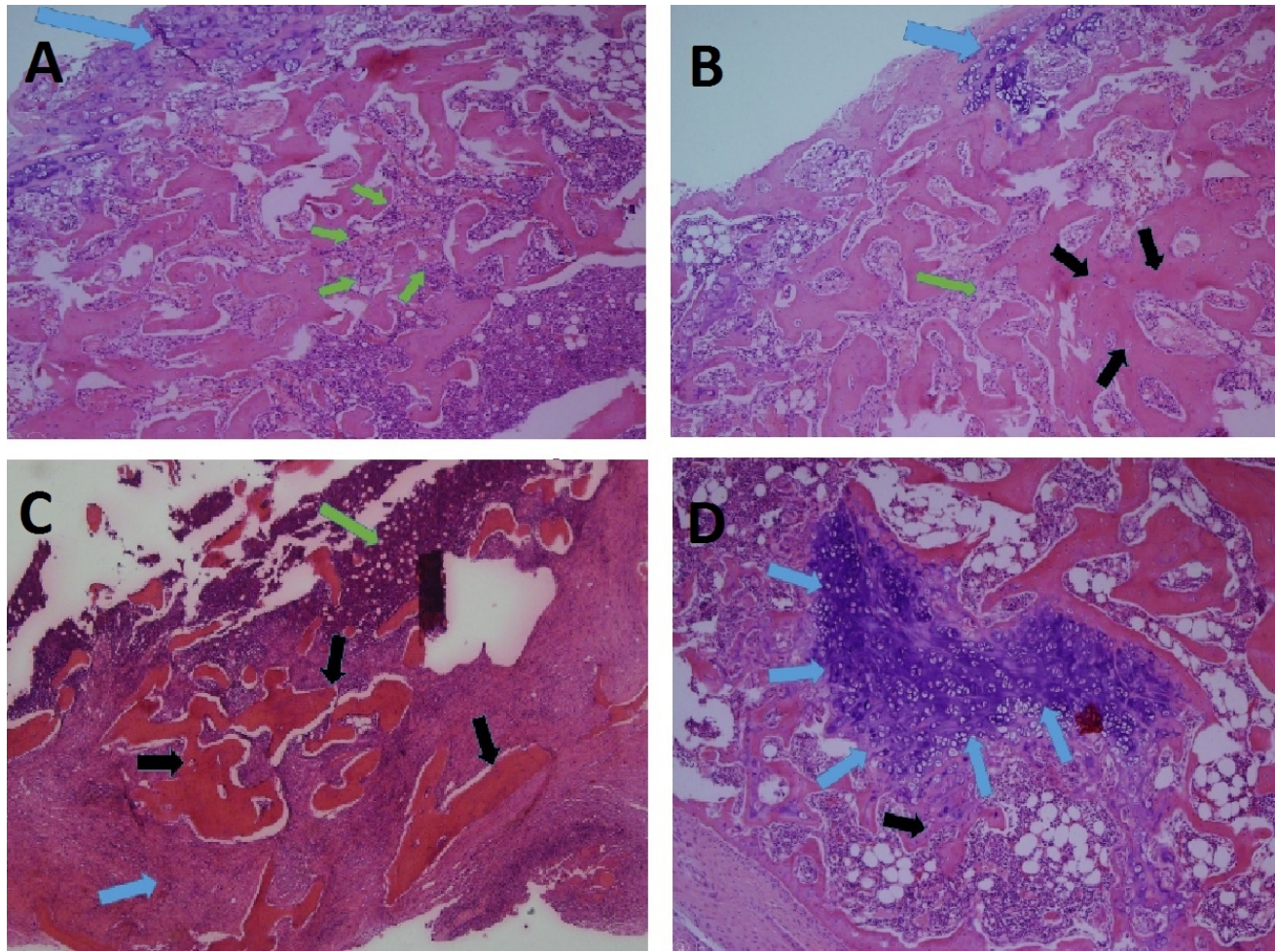

$328 \times 245 \mathrm{~mm}(96 \times 96 \mathrm{DPI})$ 


\section{TABLES}

Table 1. Radiographic scoring

\begin{tabular}{cl}
\hline Score & Criteria \\
\hline $\mathbf{0}$ & No bone mass \\
\hline $\mathbf{1}$ & Only unilateral bone mass \\
\hline $\mathbf{2}$ & Bilateral radiolucent bone mass \\
\hline $\mathbf{3}$ & Bilateral bone mass with unilateral radiolucency \\
\hline $\mathbf{4}$ & Non-radiolucent bilateral bone mass \\
\hline
\end{tabular}

Table 2. Heiple's Modified Lane and Sandhu Histological Classification

Union

None

Fibrous union

Osteochondral union

Bone union

All reorganized

Spongious

Complete resorbed, replaced by connective tissue

Most of resorbed

Partial resorbed

No osseous cellular activity

Early apposition of new bone

Active apposition of new bone

Mostly reorganized spongious

Totally reorganized spongious

Bone marrow

Dead 


\begin{tabular}{lc}
\hline New fibrous tissue & $\mathbf{3}$ \\
Two-thirds of marrow, replaced by new tissue & $\mathbf{4}$ \\
Less than one-quarter of adult marrow & $\mathbf{5}$ \\
Fatty marrow & $\mathbf{6}$ \\
Compact (cortical) & $\mathbf{0}$ \\
None & $\mathbf{1}$ \\
Begin to appear & $\mathbf{2}$ \\
Formation & $\mathbf{3}$ \\
Totally formed & 24 \\
\hline Total points & \\
\hline
\end{tabular}

Table 3. Distribution of histopathologic examination results for each group

\begin{tabular}{|c|c|c|c|c|c|}
\hline Group & Union & Spongious & Compact & Bone marrow & Total \\
\hline (n) & $\begin{array}{l}\text { Mean } \pm \\
\text { standard } \\
\text { deviation }\end{array}$ & $\begin{array}{l}\text { Mean } \pm \\
\text { standard } \\
\text { deviation }\end{array}$ & $\begin{array}{l}\text { Mean } \pm \\
\text { standard } \\
\text { deviation }\end{array}$ & $\begin{array}{l}\text { Mean } \pm \\
\text { standard } \\
\text { deviation }\end{array}$ & $\begin{array}{l}\text { Mean } \pm \\
\text { standard } \\
\text { deviation }\end{array}$ \\
\hline D0 & $2.2 \pm 0.8$ & $6.6 \pm 0.5$ & $2.6 \pm 0.5$ & $\begin{array}{c}5.8 \pm \\
0.4\end{array}$ & $17.2 \pm 1.6$ \\
\hline D1 & $3.1 \pm 0.9$ & $6.9 \pm 0.4$ & $2.7 \pm 0.5$ & $6 \pm 0$ & $18.7 \pm 1.4$ \\
\hline D10 & $1.9 \pm 0.7$ & $6.4 \pm 0.8$ & $2.4 \pm 0.5$ & $5.6 \pm 0.5$ & $16.3 \pm 2.1$ \\
\hline D100 & $2.3 \pm 0.8$ & $7.0 \pm 0.0$ & $2.6 \pm 0.5$ & $5.9 \pm 0.4$ & $17.6 \pm 1.4$ \\
\hline $\mathbf{p}^{*}$ & 0.75 & 0.19 & 0.79 & 0.25 & 0.13 \\
\hline
\end{tabular}

